corporate influences contributing to an obesogenic environment. A current focus in childhood obesity policy debates is the role that online advertising of high in fat, sugar and salt (HFSS) food and beverages plays as a driver of childhood obesity and what regulation is required. However, there is a lack of research in this area as to public acceptability of regulation of online advertising of HFSS products as a viable policy solution. This study examines the perceptions of parents and stakeholders in regulating this online environment to answer how the regulation of online advertising of HFSS products to children is viewed in the UK?

Methods Three qualitative methods were triangulated: 1) eight focus groups with parents who have children aged between five and 15 years old; 2) scoping review of 85 responses to a 2016 Committees of Advertising Practice consultation on nonbroadcast advertising to children; and 3) 11 stakeholder interviews (industry, civil society, academics and government body). Data were analysed inductively and thematically using NVivo. Results Parents reported finding it increasingly difficult to attain a healthy diet for their children, competing with a multitude of pressures, including online advertising of HFSS products. Non-industry stakeholders agreed with this perspective, whereas industry stakeholders were sceptical as to the influence online advertising had in contributing to an obesogenic environment. In terms of attitudes to regulation, two views emerged from the three data sets: 1) support for increased regulation of online advertising of HFSS products, or 2) the continuation of the current self-regulatory model. Underpinning their views were concerns as to the distribution of power within the obesogenic environment, with the majority of parents and all non-industry stakeholders describing the food and beverage industry as possessing too much power, and government and parents possessing too little. In contrast, the remaining parents and industry stakeholders argued that government possessed too much power and as such infringed on individual autonomy.

Conclusion Parents' and stakeholders' views in this study largely aligned with Beauchamp's (1976) theory on social justice versus market justice. Although not generalisable, this study offers insights into how their perspectives on the distribution of power within the obesogenic environment may have informed their views on implementing increased regulation of the online advertising environment as a viable policy solution to tackle childhood obesity.

\section{RF23 'US' AND 'THEM': IDENTIFYING THE MOST SUITABLE APPROACH TO INVOLVING PATIENTS AND HEALTHCARE PROFESSIONALS IN A CONSENSUS PROCESS TO INFORM INTERVENTION DEVELOPMENT}

E Racine*, F Riordan, E Phillip, S McHugh, P Kearney. School of Public Health, University College Cork, Cork, Ireland

\subsection{6/jech-2019-SSMabstracts. 138}

Background Intervention development is a critical first step when conducting trials or observational studies. Research suggests that involving multiple stakeholders in this process increases the likelihood of developing interventions that are acceptable, engaging, feasible and effective. However, there is little guidance for researchers on the best ways to involve multiple stakeholders in a meaningful way. The aim of this Study Within A Trial (SWAT) is to identify the most suitable approach to involve patients and healthcare professionals in a consensus process to inform the development of the Improve Diabetes Eye-screening Attendance (IDEA's) intervention.

Methods This is a qualitative study. Three meetings were held to establish consensus on the content and delivery of the intervention. Meeting included 1 patients only, meeting 2 included a combination of patients and healthcare professionals and meeting 3 included healthcare professionals only. Stakeholders were asked to agree on intervention components which target patients and general practices. Each meeting was audio recorded and field notes were taken. After the meeting, semi-structured telephone interviews were carried to explore stakeholders' experiences of taking part. Data were transcribed verbatim and managed using NVivo V12 software. Thematic analysis was performed to identify themes relating to members' experiences of taking part.

Results All three meetings put forward feasible ideas which were incorporated into the final intervention. Each meeting had ideas which were not put forward by the other groups but were incorporated into the final intervention (Meeting $1=6$ ideas, Meeting 2=2 ideas and Meeting $3=5$ ideas). Thematic analysis suggests that stakeholders in meetings 1 and 3 felt comfortable expressing their opinions and grateful that they were given the opportunity to be heard. Stakeholders in meeting 2 felt they had to bold back on their opinions as they were aware that the other stakeholder group was in the room. They also felt that their contributions were undervalued by the other stakeholder group.

Conclusion Involving patients and healthcare professionals together in a consensus process is not a suitable approach to involvement. This study will guide researchers on the most suitable approach to involve patients and healthcare professionals in a consensus process and will contribute to the evolving literature on the potential impact of involving multiple stakeholders in the intervention development process.

\section{RF24 A SYSTEMATIC REVIEW AND META-ANALYSIS OF SCHOOL-BASED EDUCATIONAL INTERVENTIONS TO IMPROVE BODY COMPOSITION IN ADOLESCENTS}

${ }^{1} \mathrm{CM}$ Jacob, ${ }^{2,3} \mathrm{PL}$ Langdon, ${ }^{2,4} \mathrm{HM}$ Inskip, ${ }^{2} \mathrm{~T}$ Morris, ${ }^{2} \mathrm{C}$ Parsons, ${ }^{1,4} \mathrm{M}$ Hanson, ${ }^{1,3} \mathrm{~K}$ WoodsTownsend, ${ }^{2,4} \mathrm{~J}$ Baird*. Institute of Developmental Sciences, Faculty of Medicine, University of Southampton, Southampton, UK; ${ }^{2}$ MRC Lifecourse Epidemiology Unit, University of Southampton, Southampton, UK; ${ }^{3}$ Southampton Education School, Faculty of Social Sciences, University of Southampton, Southampton, UK; ${ }^{4}$ NIHR Southampton Biomedical Research Centre, University Hospital Southampton NHS Foundation Trust, Southampton, UK

\subsection{6/jech-2019-SSMabstracts. 139}

Background Adolescence is a period marked by critical changes in behaviours and body composition that place adolescents at an increased risk of becoming overweight and obese. Health education in schools has the potential to improve health behaviours by encouraging critical thinking about these issues. To develop sustainable interventions to prevent obesity, it is necessary to understand whether educational interventions during adolescence are effective, and which intervention elements are associated with improvements in BMI.

We addressed the question: do school-based educational interventions improve BMI in adolescence, and what intervention features are associated with effectiveness?

Methods We carried out a systematic review and meta-analysis of published intervention studies. We searched MEDLINE, PsycINFO, CINAHL, and ERIC from 2006 to 2017. Two 
independent researchers assessed titles and abstracts, extracted data and assessed risk of bias in the included studies, following the PRISMA guidance. Review inclusion criteria were: a) health education intervention, studies conducted in schools in high-income countries that included a control group b) participants aged 10-19 years, c) studies reporting BMI/BMI Z-score at baseline and follow-up. A meta-analysis was conducted combining findings of studies presenting data on BMI z-score $(n=13)$. Heterogeneity was assessed using Cochran's $Q$ and the percentage of variability due to heterogeneity was quantified using $\mathrm{I}^{2}$.

Results Searches identified 29,174 publications, of which 312 studies papers were selected as potentially meeting inclusion criteria. Twenty-five studies met the inclusion criteria. Most $(n=18)$ were delivered by teachers in classroom settings, followed by researchers, schools nurses and students. Additional methods used in the interventions included out of class components, physical activity sessions, digital interventions and parent involvement. Eleven of the included studies were effective in improving BMI or BMI Z-score at follow-up periods from two months to two years post-intervention. Intervention features associated with effectiveness were the provision of training for teachers prior to intervention, and involvement of parents or families. Data from 13 of the 25 studies were included in a meta-analysis, using a random effects model given the high level of heterogeneity $\left(\mathrm{I}^{2}=65.1 \%\right)$. The overall pooled estimate of change in BMI Z-score in the intervention group, compared with the control group, demonstrated that BMI improved in adolescents exposed to the intervention $[\beta=-0.10,95 \%$ CI $(-0.14,-0.05) ; p<0.001]$.

Conclusion This systematic review demonstrates that schoolbased educational interventions have the potential to prevent obesity in adolescence. Mediating factors associated with effectiveness included providing training for teachers prior to the intervention and involving parents or families.

\section{Health inequalities}

\section{RF25 EFFECTS OF HOUSING FIRST APPROACHES ON HEALTH AND WELLBEING OF ADULTS WHO ARE HOMELESS OR AT RISK OF HOMELESSNESS: SYSTEMATIC REVIEW AND META-ANALYSIS OF RANDOMISED CONTROLLED TRIALS}

\footnotetext{
${ }^{1,2} \mathrm{AJ}$ Baxter*, ${ }^{2} \mathrm{EJ}$ Tweed, ${ }^{2} \mathrm{SV}$ Katekireddi, H Thomson. ${ }^{1}$ College of Medicinal, Veterinary and Life Sciences, University of Glasgow, Glasgow, UK; ${ }^{2}$ MRC/CSO Social and Public Health Sciences Unit, University of Glasgow, Glasgow, UK
}

\subsection{6/jech-2019-SSMabstracts. 140}

Background Access to housing is an important determinant of health, with homeless people having substantially increased morbidity and mortality compared to the housed population. Conventional 'Treatment First' (TF) models for tackling homelessness provide temporary accommodation conditional on adherence to services to address health needs, particularly substance use. A new policy approach aiming to end homelessness across Europe and North America, the 'Housing First' (HF) model, provides rapid housing, not conditional on abstinence from substance use. This has been noted by other reviewers as improving housing stability, but at the potential cost of removing incentives to use health services and abstain from harmful substances. Conversely, increased housing stability may lead to health improvements. We aimed to systematically review the evidence from randomised controlled trials to evaluate the effects of HF on health and well-being. Method We searched seven databases for randomised controlled trials of interventions providing rapid access to nonabstinence-contingent, permanent housing. We extracted data for the following primary outcomes: mental health; selfreported health and quality of life; substance use; non-routine use of healthcare services. Data recording housing stability was extracted as a secondary outcome. We assessed risk of bias and calculated standardised effect sizes.

Results We included four studies, all with 'high' risk of bias. The impact of HF on most short-term health outcomes was imprecisely estimated, with varying effect directions. No clear difference in substance use was seen. Intervention groups experienced fewer emergency department visits (incidence rate ratio $(\mathrm{IRR})=0.63 ; 95 \% \mathrm{CI} 0.48$ to 0.82 ), fewer hospitalisations $(\mathrm{IRR}=0.76 ; 95 \% \mathrm{CI} 0.70$ to 0.83 ) and less time spent hospitalised (standardised mean difference $(\mathrm{SMD})=-0.14$; $95 \%$ CI -0.41 to 0.14 ) than control groups. In all studies intervention participants spent more days housed $(\mathrm{SMD}=1.24$; $95 \%$ CI 0.86 to 1.62 ) and were more likely to be housed at $18-24$ months (risk ratio $=2.46$; 95\% CI 1.58 to 3.84 ).

Conclusion HF approaches successfully improve housing stability and may improve some aspects of health. Implementation of HF would likely reduce homelessness and non-routine health service use without an increase in problematic substance use. Impacts on long-term health outcomes require further investigation.

\section{RF26 QUANTIFYING INEQUALITIES IN LOOKED AFTER CHILDREN IN ENGLAND}

ETC Lai, D Taylor-Robinson, DL Bennett*. Department of Public Health and Policy, University of Liverpool, Liverpool, UK

\subsection{6/jech-2019-SSMabstracts. 141}

Background The proportion of Looked After Children (LAC) in England is rising. The high burden and consequences of child maltreatment are well documented, yet little is known about the drivers of children being taken into care in England. In order to inform policy, this analysis aimed to determine whether there were inequalities in the sustained rise in LAC in in recent years.

Methods We analysed routinely available local authority level data from 152 English upper tier local authorities between 2003-4 and 2017-18. The principal outcome was the rate of children taken into care each year. Numbers of LAC were drawn from the 'children looked after data return', submitted by local authorities to the Department of Education annually. Child population data were sourced from Office of National Statistics mid-year population estimates. Local authorities were assigned to quintiles according to their income deprivation score based on 2015 Indices of Multiple Deprivation, the main exposure of interest. Trends were analysed descriptively. Longitudinal mixed effects models were used to assess changes in social inequalities in LAC over time. Analyses were performed in $\mathrm{R}$ version 3.5.2.

Results There was a $29.8 \%$ increase in children taken into care between 2008 and 2018 in England, which was not experienced evenly across the population. In 2008 the absolute inequalities gap between most and least deprived areas was 143 children per 100,000 (95\% CI 108 to 179). Between 